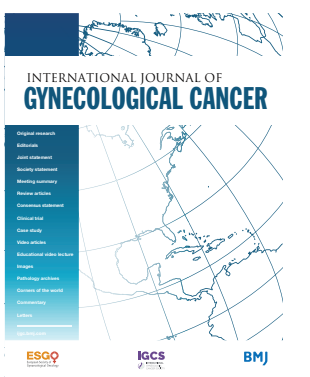

\title{
Paraneoplastic cerebellar degeneration associated with anti-Yo antibodies in uterine papillary serous carcinoma
}

\author{
Antoine Deleuze \\ Department of Medical Oncology, Centre Eugène Marquis, Rennes, France \\ Julien Farce \\ Department of Nuclear Medicine, Centre Eugène Marquis, Rennes, France \\ Constance d'Abrigeon \\ Department of Medical Oncology, Centre Eugène Marquis, Rennes, France \\ Antoine Girard \\ Department of Nuclear Medicine, Centre Eugène Marquis, Rennes, France \\ Fanny Le Du \\ Department of Medical Oncology, Centre Eugène Marquis, Rennes, France
}

\section{Correspondence to} Dr Antoine Deleuze, Department of Medical Oncology, Centre Eugène Marquis, Rennes 35042, France; a.deleuze@ rennes.unicancer.fr

Accepted 14 September 2021

Check for updates

(C) IGCS and ESGO 2022. No commercial re-use. See rights and permissions. Published by BMJ.

To cite: Deleuze A, Farce J, d'Abrigeon $\mathrm{C}$, et al. Int $\mathrm{J}$

Gynecol Cancer 2022;32:101104.

\section{CASE PRESENTATION}

A 76-year-old African American woman initially presented with a 6-month history of metrorrhagia and abdominal pain. She underwent a pelvic ultrasound, hysteroscopy, and endometrial biopsy. Initial physical examination was normal without any neurological deficits and World Health Organization Performance Status (WHO-PS) was 0. Final pathology from biopsy showed a high-grade papillary serous endometrial adenocarcinoma. A baseline serum CA125 level was $189 \mathrm{U} / \mathrm{mL}$. The staging included a fluorodeoxyglucose positron emission tomography-computed tomography (FDG-PET/CT) that showed hypermetabolic uterine, ovarian, and peritoneal lesions suggestive of carcinomatosis. Brain metabolism on FDG-PE/CT was normal. Surgical evaluation by laparoscopy confirmed limited pelvic carcinomatosis. A debulking procedure was performed including complete hysterectomy, salpingo-oophorectomy, omentectomy, sigmoid resection, and aortic and pelvic lymphadenectomy. The radical hysterectomy specimen showed a 6 $\mathrm{cm} \times 6 \mathrm{~cm}$ high-grade papillary serous carcinoma with vesico-uterine pouch, and left parametrial and ovarian compromise. Omentum and sigmoid were infiltrated by peritoneal carcinomatosis. Two of 16 lymph nodes had metastatic disease. The resection was considered complete, and the final International Federation of Gynecology and Obstetrics (FIGO) 2018 stage was IVB. Adjuvant chemotherapy was subsequently initiated, consisting of six cycles of carboplatin (AUC5 every 3 weeks) and paclitaxel $(175 \mathrm{mg} /$ $\mathrm{m}^{2}$ every 3 weeks) from July to December 2019. The post-treatment surveillance began in March 2020 with periodic clinical examination, laboratory tests, and CT scan every 3 months. In June 2020, the patient developed a mild balance disturbance with no other neurological symptoms. In September 2020, 9 months after the end of adjuvant chemotherapy, the patient presented with abdominal pain, weight loss, and persistent gait imbalance. WHO-PS was 2. Clinical examination showed extensive ascites without any pelvic abnormality. Serum CA125 was $900 \mathrm{U} / \mathrm{mL}$. FDG-PET/CT confirmed diffuse peritoneal carcinomatosis. Given her disease-free interval, the patient was treated with carboplatin AUC5 every 3 weeks and paclitaxel $80 \mathrm{mg} / \mathrm{m}^{2}$ weekly.

In February 2021, after four cycles of chemotherapy, the patient presented to the emergency department with subacute dysarthria and dizziness. She was initially admitted to the neurology service where a brain CT scan and magnetic resonance imaging (MRI) were performed and these did not reveal any structural abnormality that would explain the cerebellar symptoms. Clinical examination revealed dysarthria, horizontal gaze nystagmus, wide-based gait, truncal and appendicular ataxia predominating on the left side of the body, and fine motor control impairment. Two days later, she developed transient left arm myocIonic movements and impaired consciousness. She was subsequently transferred to the oncology service where her condition deteriorated. MRI of the brain was repeated. There was no epileptiform activity on the electroencephalogram (EEG). The most frequent etiologies of cerebellar dysfunction, including ischemic, metabolic, toxic, or infectious causes, were ruled out. Cerebrospinal fluid analysis could have provided information on cerebrospinal fluid protein, glucose levels, and cellularity but the lumbar puncture was attempted and failed 


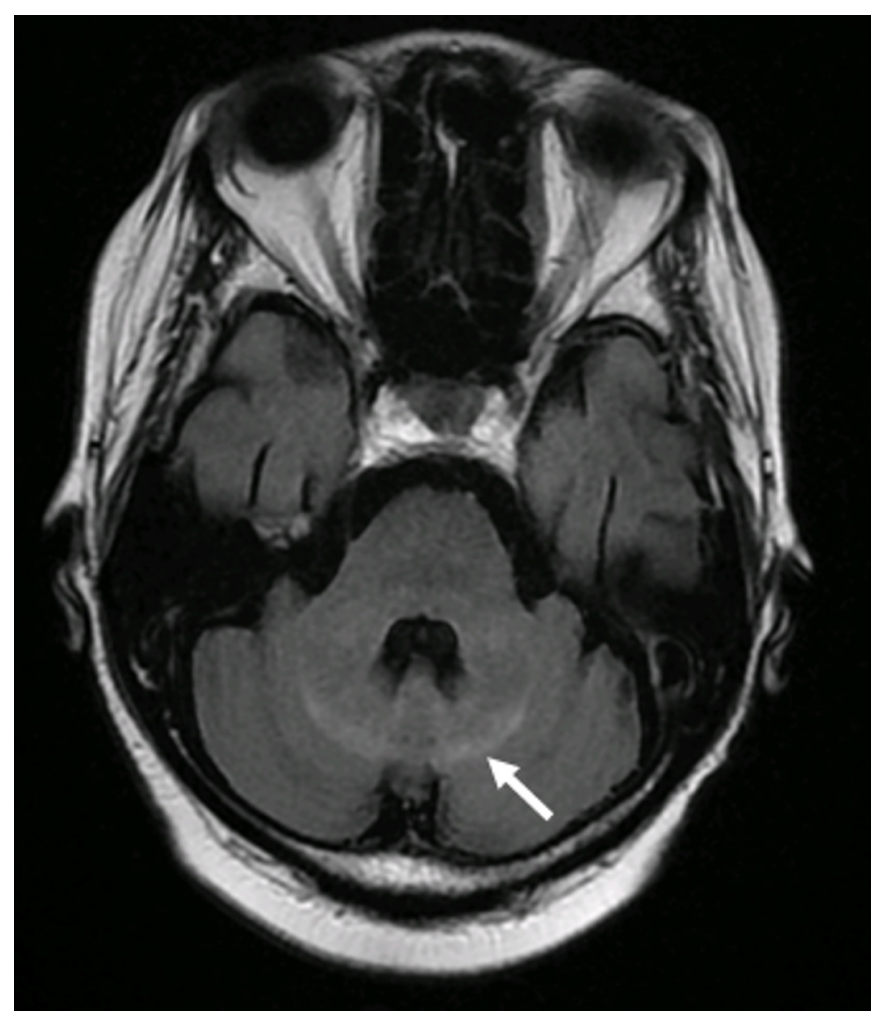

Figure 1 Brain magnetic resonance imaging, axial view of cerebellum. Hyperintense T2 fluid-attenuated inversion recovery (FLAIR) of vermis and hemispheres (white arrow).

as the patient experienced significant deterioration and could not maintain the lateral decubitus position.

MRI revealed cerebellar atrophy and an abnormal signal hyperintensity on fluid-attenuated inversion recovery (FLAIR) sequence within the vermis and cerebellar hemispheres. There was no sign of metastatic disease or ischemic events (Figure 1). The FDG-PET/CT revealed a metabolic progression of carcinomatosis in the cecum. Hypermetabolism was noted in the left cortical parieto-temporal region in association with left parietal flattened sulci most likely due to an epileptic seizure, matching the clinical findings. In contrast, a diffuse hypometabolism was noted in both frontal lobes, in the right temporal and parietal lobes, and in the left basal ganglia (Figure 2). Given the radiological findings, and the history of cerebellar symptoms manifested by ataxia, vertigo, and dysarthria, we considered

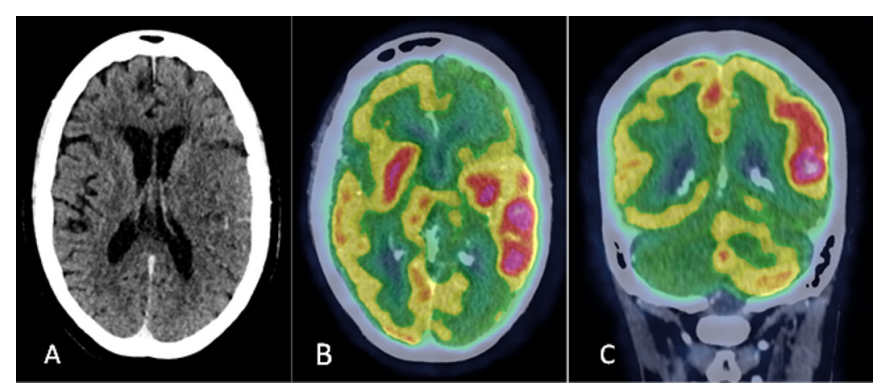

Figure 2 Left parietal flattened sulci (A). Left parietotemporal hypermetabolism and diffuse hypometabolism in both frontal lobes, in basal ganglia, and in right temporal and parietal lobes (B). Right cerebellar hypometabolism (C). both hypothesis of an associated paraneoplastic neurological syndrome associated with epilepsy as a collateral effect of leptomeningeal carcinomatosis.

Clonazepam was given at the acute phase at the dose of $2 \mathrm{mg}$ intravenous followed by levetiracetam administration of $500 \mathrm{mg}$ intravenous twice a day with a significant improvement of myoclonic movements and consciousness. Leptomeningeal carcinomatosis had been considered and cerebrospinal fluid analysis could have shown high protein level and/or cancer cells. With the hypothesis of paraneoplastic syndrome, serum onconeural antibody panel testing was performed and this was positive for anti-Yo antibodies, mostly associated with paraneoplastic cerebellar degeneration. Anti-Yo antibodies are usually detectable in the serum and rarely require cerebrospinal fluid testing, but approximately $30 \%$ of patients with suspected paraneoplastic syndrome do not have detectable antibodies in their serum. Thus, it seems reasonable to test both serum and cerebrospinal fluid to optimize the diagnosis approach.

\section{DR LE DU: WHAT IS THE EPIDEMIOLOGY AND CLINICAL SIGNIFICANCE OF PARANEOPLASTIC NEUROLOGICAL SYNDROMES?}

Among the wide array of manifestations and disabilities induced by cancer, paraneoplastic syndromes represent rare entities that do not originate from tumor growth, metabolic, or infectious complications of malignancy and its treatments. Various symptoms and conditions have been described and originate from tumor secretion of functional peptides or cytotoxic T-cells cross-reactivity phenomenon resulting in endocrine, dermatological, rheumatological, hematological, and neurological syndromes. Paraneoplastic neurological syndromes are found in less than $1 \%$ of patients with cancer, and these represent a subset of immune-mediated disorders occurring in the frame of naturally occurring antitumor immune responses. Most of the paraneoplastic neurological syndromes are diagnosed before cancer detection and present in $80 \%$ of the cases in the initial phase of the disease. Paraneoplastic cerebellar degeneration is one of the most common paraneoplastic neurological syndromes, strongly associated with small cell lung cancers, breast cancers, and gynecological cancers. Anti-Yo are the most frequently identified onconeural antibodies in paraneoplastic cerebellar degeneration and have been found to be highly specific. ${ }^{1}$ Paraneoplastic cerebellar degeneration is characterized by an acute or subacute onset of global cerebellar dysfunction including ataxia, vertigo, dysarthria, nystagmus, and involuntary movements of the head and limbs following an extensive loss of Purkinje cells mainly in vermis and midline cerebellum. ${ }^{2}$

\section{DR DELEUZE: WHAT ARE THE MAIN PHYSIOPATHOLOGICAL MECHANISMS INVOLVED IN PARANEOPLASTIC CEREBELLAR DEGENERATION?}

Paraneoplastic neurological syndromes are usually defined as classic or non-classic neurological syndromes associated with a diagnosed or undiagnosed malignancy and positivity of onconeural antibodies. Of note, several items should be mentioned to illustrate the dynamic understanding of paraneoplastic cerebellar degeneration. Pathogenic mechanisms are not fully understood but cytotoxic 
T-cells recognition of nervous system epitopes and production of neuronal autoantibodies also known as onconeural antibodies are important mechanisms. Anti-Yo antibody has been found to target intracellular cerebellar degeneration-related protein 2 (CDR2) and its ligands (CDR2L) in Purkinje cells. It is the most frequent onconeural antibody in paraneoplastic cerebellar degeneration associated with gynecological neoplasms, but up to 37 different antibodies have been described. ${ }^{3}$ However, nearly $50 \%$ of cases with paraneoplastic cerebellar degeneration remain seronegative, making their diagnosis challenging.

It has been recently described that ovarian tumors associated with anti-Yo-positive paraneoplastic cerebellar degeneration tend to present copy number variations or mutations in CDR2 and CRD2L genes resulting in the priming of neoantigens triggering initiation of immune response. Indeed, in this study, modifications in CDR2/ CDR2L sequences were associated with an important infiltration of activated CD8-T-cells, macrophages, and microglia cells. ${ }^{4}$ Interestingly, onconeural antigen-specific T-cells have been found to induce neuronal death with assistance from antigen-specific CD4 cells in animal model of paraneoplastic cerebellar degeneration, highlighting the potential key role of cytotoxic T-cells as mediators of neuronal injury. ${ }^{5}$ The pathogenic role of CDR2/CDR2L targeting by anti-Yo antibodies remains unclear. In vitro studies reported a possible neurotoxic effect of anti-Yo relying on calcium homeostasis dysfunction and resulting in cell death. However, their direct pathogenic effect has not yet been proven in animal models. ${ }^{6}$

Initial MRI is usually normal in most patients. After a few weeks of evolution, axial FLAIR images of cerebellar hyperintensities can be found, frequently associated with atrophy of the cerebellar hemispheres and vermis. ${ }^{7}$ By contrast, FDG-PET/CT has revealed, in a series of paraneoplastic cerebellar degeneration cases, various abnormalities including cerebellar hypermetabolism at the onset of neurological symptoms and diffuse cortical hypometabolism in later phases. This study might be an interesting biomarker for diagnosis, especially in patients with normal MRI or in seronegative paraneoplastic cerebellar degeneration.$^{78}$

\section{DR. LE DU: WHAT WOULD HAVE BEEN YOUR THERAPEUTIC APPROACH?}

It is now well established that neurological immune-mediated damage in paraneoplastic cerebellar degeneration is mainly irreversible. The role of cancer treatment in the evolution of neurological symptoms remains uncertain, even in cases of objective tumor response. The interest in immunosuppressive approaches in paraneoplastic cerebellar degeneration mostly relies on their efficacy in autoimmune diseases as no controlled trials or large cohort studies have been published. Rituximab, an anti-CD20 antibody, given at $375 \mathrm{mg} / \mathrm{m}^{2}$ monthly, appeared to improve the neurological condition assessed using the modified Rankin scale in nine patients including anti-Hu and anti-Yo paraneoplastic neurological syndromes. ${ }^{9}$ Similarly, a few case reports have described neurological improvement after intravenous immunoglobulin treatment with various therapeutic schemes, in association with high-dose corticosteroids. ${ }^{10}{ }^{11}$ Corticosteroids are widely prescribed, however, their efficacy in monotherapy is yet to be demonstrated. Plasmapheresis has been reported with a rapid decrease of cerebellar dysfunction in one patient, and a substantial decrease in the anti-YO antibodies titer in the serum. ${ }^{12}$

High-dose IV corticosteroids at $10 \mathrm{mg} / \mathrm{kg}$ per day were administered for 7 days and were ineffective. Neurological symptoms worsened, severe ataxia led to bed confinement, and the patient became obtunded over the course of a week. The medical team decided to stop chemotherapy and initiate best supportive care. The patient's poor functional status and prognosis required that she be discharged to a palliative care facility where she died in April 2021.

\section{CLOSING SUMMARY}

Anti-Yo-positive paraneoplastic cerebellar degeneration associated with endometrial cancer is a rare occurrence and our case presents several items that are worthy of mention. Remarkably, the patient did not show any cerebellar dysfunction at cancer diagnosis as is usually seen in paraneoplastic cerebellar syndromes. Rather, the onset of neurological disorder began after the first cancer relapse. Moreover, our patient presented intricate neurological symptoms involving symptoms of both infratentorial dysfunction (paraneoplastic cerebellar syndrome) as well as supratentorial cortical dysfunction (paraneoplastic epilepsy). FDG-PET/CT exhibited metabolic changes suggestive of left parieto-temporal epilepsy but also revealed a diffuse cortical hypometabolism of unknown origin that we hypothesized to result from paraneoplastic neurotoxic immunemediated lesions in the absence of other documented causes.

Paraneoplastic cerebellar degeneration is a rare and debilitating immune-mediated disorder associated with extensive loss of Purkinje cells and inflammatory infiltrates in the cerebellar cortex, deep cerebellar nuclei, and inferior olivary nuclei. ${ }^{13}$ In most cases, paraneoplastic cerebellar degeneration is the first manifestation of an underlying cancer characterized by rapidly evolving asymmetrical and usually bilateral neurological symptoms of trunk and appendicular ataxia, dysarthria, and nystagmus. Less frequently, paraneoplastic cerebellar degeneration may develop after cancer diagnosis and usually leads to uncontrolled or imminent disease recurrence. The most associated malignancies are ovarian cancers, breast cancers, and Hodgkin's lymphoma, and it should be highlighted that paraneoplastic cerebellar degeneration rarely originates from endometrial cancers. ${ }^{14}$ Anti-Yo antibodies are strongly associated with paraneoplastic cerebellar degeneration but their pathological effect remains uncertain, as cumulative evidence tends to highlight the prominent role of specific CD8 T-cells and immune cell infiltration. To date, the use of immunosuppressive approaches in the treatment of paraneoplastic cerebellar degeneration seem promising as neurological improvements have been observed in several patients treated with rituximab and intravenous immunoglobulins. However, data are still lacking and controlled trials are needed to evaluate the efficacy of such strategies and determine the best therapeutic option.

Contributors AD, JF, AG: critically reviewed images description. FLD: discussant. All authors contributed to the conception and writing of the manuscript.

Funding The authors have not declared a specific grant for this research from any funding agency in the public, commercial or not-for-profit sectors.

Competing interests FLD: consulting fees for Lilly; payment or honoraria for lectures, presentations, speakers bureaus, manuscript writing,or educational events for Lilly; support for attending meetings and/or travel for Pfizer, Lilly, 


\section{Case study}

Novartis; participation on a Data Safety Monitoring Board or Advisory Board for Lilly, Pfizer, Novartis, SeaGen, Daiichi.

Patient consent for publication Not applicable.

Provenance and peer review Commissioned; internally peer reviewed.

\section{REFERENCES}

1 Pelosof LC, Gerber DE. Paraneoplastic syndromes: an approach to diagnosis and treatment. Mayo Clin Proc 2010;85:838-54.

2 Vernino S. Paraneoplastic cerebellar degeneration. Handb Clin Neurol 2012;103:215-23.

3 Yshii L, Bost C, Liblau R. Immunological bases of paraneoplastic cerebellar degeneration and therapeutic implications. Front Immunol 2020;11:991.

4 Small M, Treilleux I, Couillault C, et al. Genetic alterations and tumor immune attack in Yo paraneoplastic cerebellar degeneration. Acta Neuropathol 2018;135:569-79.

5 Gebauer C, Pignolet B, Yshii L, et al. CD4+ and CD8+ T cells are both needed to induce paraneoplastic neurological disease in a mouse model. Oncoimmunology 2016;6:e1260212.

6 Galli J, Greenlee J. Paraneoplastic diseases of the central nervous system. F1000Res 2020;9. doi:10.12688/f1000research.21309.1. [Epub ahead of print: 0603 2020].
7 Choi K-D, Kim JS, Park S-H, et al. Cerebellar hypermetabolism in paraneoplastic cerebellar degeneration. J Neurol Neurosurg Psychiatry 2006;77:525-8.

8 Abdulaziz ATA, Yu XQ, Zhang L, et al. Paraneoplastic cerebellar degeneration associated with cerebellar hypermetabolism: case report. Medicine 2018;97:e10717.

9 Shams'ili S, de Beukelaar J, Gratama JW, et al. An uncontrolled trial of rituximab for antibody associated paraneoplastic neurological syndromes. J Neurol 2006;253:16-20.

10 Schessl J, Schuberth M, Reilich P, et al. Long-term efficiency of intravenously administered immunoglobulin in anti-Yo syndrome with paraneoplastic cerebellar degeneration. J Neurol 2011;258:946-7.

11 Phuphanich S, Brock C. Neurologic improvement after high-dose intravenous immunoglobulin therapy in patients with paraneoplastic cerebellar degeneration associated with anti-Purkinje cell antibody. $J$ Neurooncol 2007;81:67-9.

12 Meloni C, lani C, Dominijanni S, et al. A case report of plasma exchange therapy in non-paraneoplastic cerebellar ataxia associated with anti-Yo antibody. Ther Apher Dial 2004;8:500-2.

13 Dalmau J, Rosenfeld MR. Paraneoplastic syndromes of the CNS. Lancet Neurol 2008;7:327-40.

14 Karpathiou G, Da Cruz V, Casteillo F, et al. Paraneoplastic cerebellar degeneration and endometrial cancer: a rare occurrence. Pathology 2016;48:275-8. 